\title{
The Prioritization of Risk Reducing Measures in View of Uncertain Cost/Benefits
}

\author{
Thor Bjørkvoll, Helge Langseth \\ SINTEF Industrial Management, N-7465 Trondheim, NORWAY and \\ Norwegian University of Science and Technology. N-7465 Trondheim, NORWAY \\ Email: thor.bjorkvoll@sintef.no
}

\begin{abstract}
This paper discusses the traditional cost-benefit analysis in the context of prioritization of risk reducing measures. Such measures are often uncertain both in terms of cost of the measures and their effects. We introduce the concept of decision flexibility to develop an alternative framework for making such decisions.
\end{abstract}

\section{Introduction}

To improve the Health, Safety and Environmental (HSE) situation, a decision-maker is typically confronted with a set of possible measures (projects or actions) targeted at reducing the risk. Based on some criteria, one of the measures is then selected and implemented. This paper's point of departure is the traditional cost-benefit analysis. In this setting, each measure is examined to reveal its implementation cost, as well as its benefits (e.g., in terms of the calculated risk reduction). The cost and benefit are described as single numerical values, the cost-benefit ratio is calculated, and the measure with the lowest ratio (lowest cost per unit of benefit) is given first priority. This approach totally ignores the uncertainty in costs and benefits, as only the mean values for these quantities are used.

In real-life situations both the measures' cost and risk reduction abilities can be unknown at the time when the decision is made. They should therefore be treated as random variables. In this paper we consider the decision problem not as an once-ina-lifetime decision, but rather as a decision process. The reasoning behind this is that after a measure is implemented, we may receive information regarding implementation expenditures as well as the risk reduction. We can use this information to update our beliefs in the measure's cost and benefit (e.g., following a Bayesian procedure), and it is obviously rational to let the updated beliefs form the basis for deciding whether or not a good decision was made initially. In general terms, the objective is to exploit the uncertainty in the decision problem by taking maximal advantage of the "upside" of the uncertainty involved whereas the "downside" is minimized. We will focus attention to the situation where the measures' costs are uncertain whereas their effects are considered known; similar ideas as we employ here can also be used when the risk reduction capabilities are uncertain. 


\section{An example}

We introduce our way of thinking through a simple example originally prepared for a helicopter transportation safety study [1]. There are two alternative measures $\mathrm{C}$ and $\mathrm{U}$ designed to improve safety. The benefit of each measure is set equal to the percentage of expected reduction of casualties per year. Both measures incur operating expenditures only. To keep it simple, we assume that there is no uncertainty associated with the effect (benefit) of the risk reducing measures. Both measures $\mathrm{C}$ and $\mathrm{U}$ will reduce the expected number of casualties with $5 \%$.

While the annual operating expenditures for $\mathrm{C}$ are fixed and well known, the corresponding value for $\mathrm{U}$ is uncertain. The annual operating expenditures for $\mathrm{C}$ are $€ 200.000$, hence the annual cost per percentage risk reduction is equal to $€ 40.000$. The "uncertain" measure $U$ will induce annual operating expenditures equal to either $€ 100.000$ (each and every year) or $€ 400.000$ (each and every year). Both outcomes are assumed to be equally likely. This gives expected annual expenditures of $€ 250.000$, and the expected annual cost per percentage risk reduction is $€ 50.000$. Based on the mean values, a naïve application of the cost-benefit rate selects the "certain" measure C. However, this may not be the optimal solution. Assume that U is implemented, and its true annual expenditures are revealed within one year. If the expenditures prove to be high, $\mathrm{U}$ is terminated, and measure $\mathrm{C}$ is initiated instead. If the expenditures prove to be low, $\mathrm{U}$ is carried on. Note that if $\mathrm{U}$ turns out to be a low cost measure, we will save $€ 100.000$ compared to $C$ every year. If $U$ comes with a high cost, we can terminate it. In that case we will make a loss equal to $€ 400.000$ - $€ 200.000=€ 200.000$. This loss is limited to the first year. It is evident that option $U$ should be implemented if the measure's lifetime is more than two years. Although the naïve expected cost is in favour of $\mathrm{C}$, choosing measure $\mathrm{U}$ can lead to major savings. Hence, it may be favourable to "take a gamble" and implement measures to learn about their costs and safety effects. Later in the paper we shall return to this example to establish formulas for exactly when it is beneficial to go for the "uncertain" measure.

\section{Uncertainty and costs}

In this section we flesh out the ideas of the example in the previous section. We establish a precise notation for the cost structure, and derive formulas for optimal decisions. An important part of the cost structure is made up by sunk costs: investments that are made, cannot be recovered, and have no alternative use. Examples include cost of adaptation, training, custom-made equipment, etc. The model also comprises termination cost, including the cost of switching from one measure to another. We are still faced with the simple situation where we have only two measures to consider; one of these has uncertain operating expenditures whereas the other is "certain".

Table 1 summarizes relevant information about the two measures. Although simple, the model gives some insights into why it sometimes is beneficial not to follow the cost/benefit ratio when prioritizing measures. 


\begin{tabular}{|c|c|c|c|}
\hline & $\begin{array}{l}\text { Measure C } \\
\text { ("Certain") }\end{array}$ & $\begin{array}{r}\mathrm{M} \\
\text { (Two pos }\end{array}$ & $\begin{array}{l}\text { e U } \\
\text { outcomes) }\end{array}$ \\
\hline \multirow{3}{*}{$\begin{array}{l}\text { Start-up cost } \\
\text { Termination cost }\end{array}$} & \multirow{3}{*}{$\begin{array}{l}I_{C} \\
S_{C}\end{array}$} & \multirow{2}{*}{\multicolumn{2}{|c|}{$\begin{array}{l}I_{U} \\
S_{U}\end{array}$}} \\
\hline & & & \\
\hline & & "Low" & "High" \\
\hline $\begin{array}{l}\text { Operating expenditure } \\
\text { A priori belief }\end{array}$ & $\begin{array}{c}d_{C} \\
N / A\end{array}$ & $\begin{array}{c}d_{U L} \\
p_{L}\end{array}$ & $\begin{array}{c}d_{U H} \\
p_{H}=1-p_{L}\end{array}$ \\
\hline
\end{tabular}

Table 1. Parameters describing measures $\mathrm{C}$ and $\mathrm{U}$

At the point of making the decision, the operating expenditure (opex) of measure $U$ is not known; it is either "high" with value $d_{U H}$ or "low" with value $d_{U L}{ }^{1}$. Our a priori belief in having "high" operating expenditure is $p_{H}$; the a priori belief in having "low" expenditure is $p_{L}$, where $p_{H}+p_{L}=1$.

We assume that exactly one of the measures must be implemented and operating at any time. As the two measures offer the same risk reduction, the decision maker only has to minimize the net present value of all relevant costs. To simplify we assume a fixed cost of capital, or interest rate $r$ per period, and an infinite horizon.

The information revelation process is simple: We will not learn anything about the true operating expenditures until a predefined time $t_{r}$, when the true value is uncovered. Note that this information revelation scheme makes only some decision scenarios eligible:

- If we decide to start with measure $\mathrm{C}$, then we will not learn anything about $\mathrm{U}$, and therefore never get any incentive to start $\mathrm{U}$.

- If we start with choosing measure $\mathrm{U}$, then there is no reason to terminate it before $t_{r}$. At that time, we have a fully informed decision problem, and will chose either to terminate $U$ (and instead start $C$ ) or continue $U$. It will not be optimal to revert the decision made at $t_{r}$ at any later time.

Implementing the sure measure $\mathrm{C}$ forever will induce a start-up costs $I_{C}$ and annual operating expenditures equal to $d_{C}$. For capital cost $r$, the net present value of all relevant expenditures over an infinite time horizon will be:

$$
N P V_{C}(\infty)=\frac{d_{C}}{r}+I_{C}
$$

Implementing and operating the uncertain measure over infinite time yields the following expected net present value:

$$
E\left(N P V_{U}(\infty)\right)=p_{H}\left(\frac{d_{U H}}{r}\right)+p_{L}\left(\frac{d_{U L}}{r}\right)+I_{U}
$$

\footnotetext{
${ }^{1}$ To avoid trivial cases, we shall assume that if $U$ takes the low cost, then this is beneficial to selecting measure $\mathrm{C}$. On the other hand, if $\mathrm{U}$ is known to take on its high cost, then selecting $\mathrm{C}$ will be beneficial. If these requirements are not met, it means that either $\mathrm{U}$ dominates $\mathrm{C}$ or vice versa; i.e., an uninteresting decision problem
} 
If the decision maker is limited to consider only these two alternatives, she should implement the measure $\mathrm{C}$ if and only if $N P V_{\mathrm{C}}(\infty)<\mathrm{E}\left(N P V_{\mathrm{U}}(\infty)\right)$. However, as indicated by the introductory example, it may be beneficial to run the uncertain measure until we have adequate information about costs, which will occur at time $t_{r}$. Implementing and operating the uncertain measure for the time period up to $t_{r}$, and terminating it, yields the following net present value if the operating cost of $U$ proves to be high:

$N P V_{U_{H}}\left(t_{r}\right)=\left(\frac{d_{U H}}{r}\right)\left(1-e^{-r t_{r}}\right)+I_{U}+S_{U} e^{-r t_{r}}$

For the low cost scenario it will be beneficial to operate $U$ forever, and an expression similar to Equation 1 will apply.

An interesting case emerges when the expected net present value of expenditures for running the uncertain measure forever is greater than running the certain option forever. In this case, it may nevertheless be beneficial to start running measure $U$, and then terminate it if the costs prove to be high (and hence switch to the certainty option). This happens exactly when:

$\left(\frac{d_{U H}}{r}\right)+S_{U}<\left(\frac{d_{C}}{r}\right)+I_{C}$

In cases where Equation 2 apply, we can interpret the certain measure $\mathrm{C}$ as an insurance against high cost outcomes. Hence, Equation 2, which expresses the switching possibility, is crucial for our framework.

If switching is profitable, the expected net present value of implementing the uncertain measure will be:

$E\left(N P V_{U / C}\left(t_{r}, \infty\right)\right)=$

$p_{H}\left[\left(\frac{d_{U H}}{r}\right)\left(1-e^{-r t_{r}}\right)+I_{U}+S_{U} e^{-t_{r}}+\left(\frac{d_{C}}{r}+I_{c}\right) e^{-r t_{r}}\right]+p_{L}\left[\left(\frac{d_{U L}}{r}\right)+I_{U}\right]$

The expected gain $E(G)$ of implementing the uncertain measure compared to implementing the certain measure is $E(G)=N P V_{C}(\infty)-E\left(N P V_{U \mid C}\left(t_{r}, \infty\right)\right)$, which can be stated as

$$
E(G)=\left[N P V_{C}(\infty)-E\left(N P V_{U}(\infty)\right)\right]+p_{H}\left[\left(\frac{d_{U H}}{r}-\frac{d_{C}}{r}\right)-\left(S_{U}+I_{C}\right)\right] e^{-r t_{r}}
$$

The optimal decision rule is to implement $U$ whenever $E(G)>0$. The first bracket of Equation 3 is the relevant criterion for a "once for all"-decision. The expected value of the switching option is given by the second bracket. For some cases, the value of the first brackets is negative, while the second bracket is so large that it makes $E(G)$ positive. This is exactly when the switching option makes measure $U$ preferable to measure $C$, although $C$ appears best in calculation based only on expected values.

The value of the switching option is related to the avoidance of the high cost outcome $d_{U H}$ and the opportunity cost $d_{C}$. Equation 3 demonstrates that sunk costs, represented by start-up and termination costs, reduces the gain from switching. Hence, they represent economic impediments to our getting profit from learning, and hence cause so-called lock-in effects. It is straight forward to see that the 
discount factor $r$ makes sure that the gain from the switching option is reduced as the time to cost revelation $t_{r}$ is increased. ${ }^{2}$

Equation 3 also demonstrates how an increased cost spread is favourable as the gain arises from avoidance of the high cost outcome. This can easily be demonstrated by a minor adjustment of the introductory example. With a cost of capital equal to $r=10 \%$ per period, the net present value for the "certain" measure $\mathrm{C}$ is $€ 2.000 .000$. By implementing the uncertain measure, $\mathrm{U}$, the expected net present value is reduced to $€ 1.590 .909$. We now change the values of the $d_{U H}$ and $d_{U L}$ without altering $E\left(N P V_{U}(\infty)\right)$ : We raise the high cost outcome from $d_{U H}=$ $€ 400.000$ to $d_{U H}=€ 450.000$, and reduce the low cost outcome from $d_{U L}=€ 100.000$ to $d_{U L}=€ 50.000$. We are able to take full advantage of the low cost outcome, and at the same time we are almost insulated from the high cost outcome, so the net present vale decreases further to $€ 1.363 .636$. Indeed, the measures expected value does not reflect its full potential for cost saving.

The problem described and solved above has a learning phase of length $t_{r}$. During this phase one has to pay the operating cost. Hence this phase can be characterized as a pay-as-you-go exploration with a possibility of terminating the specific measure if it turns out to be unfavourable. The pay-as-you-go property makes the problem difficult to solve for several measures even without introducing gradual information revelation of cost and effect and the possibility of parallel implementation of several measures ${ }^{3}$, see for example Weitzman [4], Gittins and Jones[5], Banks and Sundaram [7] and Sundaram [8] for contributions on search economics. Weitzman [4] separates the learning phase from the implementation phase, while the others study the optimal acquisition of information by economic agents under different cost assumptions. Indeed, these contributions demonstrate that in order to obtain analytical decision rules, the assumptions must be "simple" with respect to cost structures and/or information revelation.

\section{Summary}

In this paper we have developed a framework for prioritization of risk reducing measures when costs are uncertain. The framework rests on the definition of decision flexibility, and utilizes this flexibility as a kind of an "insurance policy" towards undesired outcomes.

The concept that is of most importance in this respect is the economic reversibility of the available measures. A measure is said to be reversible if it $i$ ) can be changed or terminated at only limited costs and ii) most of the investments undertaken can be recovered. In contrast, an economic irreversible measure

${ }^{2}$ This is a consequence of assuming that we get perfect information at $t_{r}$ no matter how small $t_{r}$ is. A more realistic information revelation scheme, where information gradually is obtained, is however outside the scope of this paper.

${ }^{3}$ As long as there is a start-up cost, the termination cost itself does not necessarily cause additional problems. If the termination cost is fixed and depends only on which measure one switch from, then it can be incorporated in the investment cost by increasing investment with the amount $S_{U}$ and simultaneously add an annual reward equal to the rent of $r S_{U}$. It is straight forward to show that the net present value of these two cash flows are equivalent and independent on when switching takes place. 
typically requires specialized investments upfront with no alternative use. If a measure is reversible, then the uncertainty wrt its cost and benefit is really a blessing in disguise: If new information point towards the underlying ("true") cost/benefit ratio being higher than assumed, we may simply terminate the measure. If new information points towards a lower cost/benefit ratio than initially expected, then the measure is continued. In general, the cost structure must be explicitly taken into account. Typically some costs will be sunk, and there may be costs connected to the transition from one measure to another (for example due to organizational effects and changes). Furthermore, the cost of gathering information, the time period needed to terminate the measure, and the relevant cost of capital should also be taken into account.

A prerequisite of our approach is the possibility of revelation of relevant information. Monitoring the accrued costs can be done with high accuracy, but revealing information regarding safety effects may be difficult or even impossible if the measure is intended to prevent accidents that occur very infrequently. Furthermore, information is revealed only gradually in these cases, and indirect measurements through indicators of different kinds will most likely have to be utilized.

\section{References:}

1. NOU 2002:17: Helicopter Safety on the Norwegian Continental Shelf - Review 2: Trends, objectives, risk influencing factors and measures.

2. Aven, Terje Foundations of Risk Analysis. New York, Wiley 2003

3. DeGroot, Morris H:Optimal statistical decisions, New York, McGraw-Hill 1970

4. Weitzman, Martin L.:Optimal Search for the Best Alternative, Econometrica, Vol 47, 1979, 641-654

5. Gittins, J. and D. Jones: Dynamic Allocation Index for the Sequential Allocation of Experiments, in (J. Gani, et al, Eds.) Progress in Statistics, North Holland, Amsterdam. 1974

6. Banks, J.S. and Rangarajan.K. Sundaram Switching Costs and the Gittins Index, 1994 Econometrica 62(3), 687-694.

7. Sundaram, Rangarajan K. Generalized Bandit Problems," Working Paper, Stern School of Business; forthcoming in Social and Strategic Behavior: Essays in Honor of Jeffrey S. Banks (David Austen-Smith and John Duggan, Eds). 2003

\section{Acknowledgements}

Terje Aven and Per Hokstad commented on an early draft of this paper. 
